\title{
Construcción de significados sobre liderazgo y su contribución a la superación de la pobreza extrema
}

\author{
Lina Alejandra Betancur Serna ${ }^{1}$ \\ Ertilia Herrera Herrera ${ }^{2}$
}

\section{RESUMEN}

El presente artículo está centrado en comprender la construcción del significado de liderazgo de las Madres Líderes de la Comuna 1-Medellín y su contribución a la superación de la pobreza extrema en el marco del Programa Medellín Solidaria-Más Familias en Acción. En este sentido, se plantearon preguntas relacionadas con la construcción del propio liderazgo, los actores inmersos y la contribución realizada a la superación de la pobreza, apuntando a la generación de nuevas reflexiones mediante las cuales fuera posible fortalecer las acciones de liderazgo en la ciudad de Medellín. El abordaje metodológico se realizó desde la investigación cualitativa y desde una perspectiva funcionalista, cuyo eje transversal es el enfoque de las capacidades; la estrategia metodológica se llevó a cabo desde el estudio de caso cualitativo y la interpretación de los datos se realizó atendiendo los principios de la etnometodología y la fenomenología, constituyéndose en meta-teorías para la comprensión del lenguaje y los significados construidos por las líderes. Los resultados obtenidos evidencian el liderazgo como una práctica social que posibilita direccionar procesos, movilizar, promover acciones orientadas a la identificación de recursos y potencialidades que proporcionen la construcción de mejores condiciones de vida y el fortalecimiento del tejido social.

Palabras claves: Liderazgo, Significados, Pobreza, Capacidades.

Recibido: 12 de septiembre de 2014

Aceptado: 10 de marzo de 2015

1 Psicóloga egresada de la Universidad de San Buenaventura, Medellín - Colombia/Magister en Gestión del Desarrollo Comunitario UNAN-Managua. Correo Electrónico: linabetancurserna@gmail.com

2 Master en Planificación ambiental y gestión de recursos naturales, especialista en gestión del desarrollo comunitario y en docencia universitaria. Correo Electrónico: eherrerah06@yahoo.es 


\title{
Construction of meanings on leadership and its contribution to overcoming extreme poverty
}

\begin{abstract}
This article is focused on understanding the construction of the meaning of leadership of Leader Mothers in the Commune 1-Medellín and their contribution to overcoming extreme poverty within the Medellín Solidarity Program-More Families in Action. In this sense, questions related to building leadership itself, the players involved and the contribution to overcoming poverty, aiming at generating new insights by which it was possible to strengthen the actions of leadership in the city Medellín were raised. The methodological approach was made from qualitative research and from a functionalist perspective, the transverse axis is the capability approach; the methodological strategy was conducted from qualitative case study and the interpretation of the data was carried out according to the principles of ethnomethodology and phenomenology, becoming meta-theories for understanding the language and meanings constructed by the leaders. The results show leadership as a social practice that allows addressing processes, mobilizing, promoting actions to identify resources and potentials to provide better living conditions and the strengthening of the social thread.
\end{abstract}

Keywords: Leadership, Meanings, Poverty, Capacities. 


\section{INTRODUCCIÓN}

Medellín, considerada una de las principales ciudades de Colombia, ha buscado articularse a las iniciativas nacionales relacionadas con el mejoramiento de las condiciones de vida de los hogares, en especial de aquellos que se encuentra en situación de pobreza, promoviendo a su vez una ciudad más solidaria y equitativa. Por ello, uno de los programas de la Alcaldía Municipal que busca mejorar la calidad de vida de las familias y garantizar el acceso integral y sostenible de los hogares más vulnerables es el Programa "Medellín Solidaria", el cual ha creado alianzas estratégicas a nivel nacional con la Red Unidos y el Programa Más Familias en Acción: la primera, orientada a que las familias alcancen los logros correspondientes a las dimensiones del Desarrollo Humano y, la segunda, foco de interés de la investigación, orientada a una entrega de transferencias monetarias condicionadas para los menores de 18 años, buscando promover la asistencia y permanencia en la escuela, así como impulsar el desarrollo integral de ellos/as, fortaleciendo el capital social mediante procesos de encuentro e integración comunitaria. De ésta manera, se parte de la premisa que las familias son sujetos activos, responsables ante la sociedad y que de ellos dependerá mejorar las condiciones de vida de sus hogares.

Dentro de la estrategia del programa, existen dos figuras del proceso: los/as titulares y los/as líderes: la primera de ellas, es la encargada de representar a su grupo familiar y es la responsable de que sus hijos cumplan con los compromisos adquiridos y, la segunda corresponde a una de las personas titulares del programa quién es elegida como líder por los demás beneficiarios para que por dos años lideren acciones encaminadas a fortalecer el bienestar comunitario y a su vez apoyar la buena marcha del programa. En relación a esto, el programa afirma: "La madre líder es una figura que encabeza procesos de participación social y concertación institucional con las madres de su entorno" (Manual Operativo Programa Más Familias en Acción 2013 (p. 17)).
A nivel general, las líderes del programa son mujeres amas de casa y en su mayoría este rol representa para ellas su primer ejercicio en lo público; es así, como su liderazgo se ha ido configurando con unas prácticas propias que les ha permitido posicionar su labor en la comunidad como una persona de referencia que se interesa por comprender las necesidades de las personas, llevándolas a avanzar en la gestión de alternativas para solucionar sus problemas, identificar los recursos con los que cuenta y fomentar un trabajo conjunto desde la construcción de lazos para el fortalecimiento del tejido social.

A raíz de las consideraciones anteriores, surgieron reflexiones relacionadas con dicha figura de liderazgo, las cuales aludieron en un primer momento sobre la inquietud por comprender ¿Cómo se realizado la construcción de su propio liderazgo? ¿Cuáles han sido los factores que están inmersos en dicha construcción? ¿Cuál es la contribución de las líderes hacia la superación de la pobreza extrema?, sumado también a ¿Cuáles son las transformaciones que estos han posibilitado en sus sectores?, donde están de por medio las demandas de los beneficiarios, las instituciones y el mismo programa en relación a su labor. De esta manera, el interés estuvo enfocado hacia la construcción de significados desde la experiencia de las líderes respecto a su rol, a fin de poder identificar sus aportes en relación a la superación de la pobreza extrema en el marco del Programa Medellín Solidaria - Más Familias en Acción.

\section{MATERIALES Y MÉTODOS}

\section{EI Estudio de caso como Estrategia Metodológica}

La estrategia metodológica se realizó a partir del estudio de caso cualitativo, el cual se enfoca en la particularidad, busca contribuir a una comprensión del sujeto y su contexto y permite ahondar en la construcción de nuevos significados. Esto requiere precisar el contexto 
en el cual ocurre el fenómeno, que para este caso se centró en la comuna 1-Popular; además de delimitar el tamaño de la unidad social el cual correspondió al grupo de Madres líderes pertenecientes al programa Más Familias en Acción de la Comuna 1- Popular de la ciudad de Medellín.

\section{Muestra}

El objetivo del muestreo cualitativo es comprender el fenómeno de interés, para lo cual la investigadora trabajó sobre muestras seleccionadas intencionalmente; por lo tanto, ésta fue inductiva y basada en criterios de participación y vínculos directos con el liderazgo, en donde a partir de una revisión documental y la observación directa se seleccionaron 4 madres líderes del Programa Medellín Solidaria - Más Familias en Acción pertenecientes a la Comuna 1 - Popular, con proyección reconocida en la comunidad y con experiencia en el ejercicio de su rol.

\section{La investigación cualitativa como abordaje metodológico}

El abordaje metodológico fue desde la investigación cualitativa, la cual aborda realidades subjetivas e intersubjetivas, lo que permite tener un contacto directo con los diversos actores y escenarios, apartando prejuicios y creencias. En así, como se estudió a las Madres líderes desde su contexto y de las situaciones en las que se hallaban, interactuando con ellas de un modo natural, tratando de comprenderlas desde su marco de referencia y experimentando la realidad desde ellas.

\section{Etnometodología y fenomenología como meta-teoría para la comprensión del lenguaje y los significados}

El enfoque de la investigación fue la etnometodología, la cual se interesa por la intersubjetividad, la acción social y el lenguaje en su cotidianidad; así mismo, con la mirada fenomenológica se pudo volver sobre la intuición reflexiva, acercándose a la comprensión de la realidad tal y como se presenta en la conciencia del actor. Desde la investigación, se buscó realizar una descripción de la experiencia y la manera de vivirla las líderes sin preconcepciones ni prejuicios, sino poniendo entre paréntesis el conocimiento propio sobre el mundo, basándose en la subjetividad trascendental, orientada hacia el ser del mundo y la conceptualización de la incertidumbre.

\section{Método Etnográfico}

$\mathrm{Su}$ principal objetivo radica en comprender una determinada forma de vida desde el punto de vista de quienes pertenecen de manera natural a esta. En éste, la Observación Participante tiene especial privilegio, en tanto desde la perspectiva de Kawullich, B (2005) citando a Marshall y Rossman (1989) posibilita hacer una "descripción sistemática de eventos, comportamientos y artefactos en el escenario social elegido para ser estudiado". En la investigación, se hizo uso tanto de la observación como de la participación de los procesos comunitarios con el fin de recolectar datos a partir de los mismos y de la interacción con las líderes; en ésta, se tuvo en cuenta la cotidianidad y vida de las mismas, a fin de fomentar el entendimiento del contexto y recogiendo datos cualitativos para ser analizados posteriormente.

\section{Métodos Grupales}

Los grupos nominales son una estrategia que posibilita obtener información de una manera estructurada y en donde se aprovecha el conocimiento y la experiencia de cada una de las personas. Bajo esta lógica, se realizaron 2 grupos nominales que contaron en total con una participación de 56 madres líderes de la comuna 1- Popular, cuyo objetivo estuvo centrado en comprender el significado construido por ellas sobre su rol a partir de sus experiencias. 


\section{Entrevistas semi-estructuradas con informantes claves}

Se basa en una conversación con un propósito definido que permita reunir datos a través de diversas preguntas. Es así, como la entrevista posibilitó entablar discusiones abiertas y realizar preguntas en profundidad, a partir de una comunicación directa con las madres líderes.

\section{Revisión de fuentes secundarias}

Las fuentes secundarias estuvieron constituidas por informes mensuales y anuales, memorias de talleres, evaluaciones, sistematizaciones, entrevistas realizadas antes del proceso y por otras personas, lo que fue necesario considerando la importancia de conocer contextos, antecedentes e informaciones que no podían ser obtenidas a través del trabajo de campo.

\section{Análisis de contenido}

El análisis de contenido fue utilizado como una técnica para realizar codificación y categorización de la información que surgió de las fuentes primarias y secundarias. Con esto, se estableció un proceso de discusión teniendo en cuenta los hallazgos recogidos durante el trabajo de campo, el referente teórico establecido y los objetivos.

\section{RESULTADOS Y DISCUSIÓN}

El estudio del tema del liderazgo en el ámbito comunitario es uno de los fenómenos más interesantes en la actualidad en tanto se dirige a la comunidad y se realiza en ambientes naturales en donde no es posible controlar comportamientos de ninguno de los miembros de la misma, ni situaciones de la realidad social en las cuales se producen procesos de liderazgo. Las razones para asumir éste rol pueden ser muchas, encontrándose para el caso de las líderes que hicieron parte de la muestra básicamente dos: la primera, por un proceso de elección democrático a partir del cual su comunidad identificó en ellas ciertas características $\mathrm{y}$ potencialidades para el trabajo comunitario $\mathrm{y}$, la segunda, por las expectativas personales frente a lo que se podrían encontrar y a los conocimientos que podrían adquirir a partir de su vinculación en el proceso.

Insertarse en liderazgo independientemente de las razones, ha permitido a las líderes adquirir capacidades y ampliar su visión, pasando del yo - tu a un "nosotros" como oportunidad y posibilidad de construcción de sociedad. Esto significa que cuando se es líder, debe haber mayor apertura y flexibilidad para trabajar y relacionarse con los otros, para poner las habilidades propias al servicio de los demás, para tener una visión compartida y para actuar en conjunto, desarrollando la conciencia de que su labor es parte de un movimiento colectivo en el que si bien saben cuál es su rol, reconocen, fomentan y buscan la participación de los otros: nosotros. Es así, como inmersas en el liderazgo ha permitido a las líderes desarrollar capacidades, definidas por Nussbaum, M y citada por Gough (2008) como el "conjunto de funcionamientos que son factibles para una persona, pudiendo elegir" (p.3), lo que supone de entrada que bajo ciertas condiciones las personas son capaces de ser y de hacer, lo cual además de ser valioso intrínsecamente, también aporta al bienestar.

En relación a los significados que las líderes han construido de su rol, a través del trabajo de campo se encontró que ser líder les ha permitido fortalecerse en la medida que han conquistado espacios públicos, vencer miedos, hacer frente a los obstáculos de una manera asertiva, salir de sus hogares, comprometerse por el mejoramiento de las condiciones de vida, aumentar su valía personal, potenciar sus capacidades, fortalecer sus autoesquemas y valorar los escenarios colectivos, en donde es necesario que la comunidad asuma un papel activo y haya un sentimiento colectivo por encima del individual que posibilite que los resultados sean mayores cuando el trabajo involucra a todos. En ésta perspectiva, Montero (2006) menciona que: 
"Se habla de solidaridad comunitaria, de necesidades compartidas, de historia común, de objetivos comunes, (...). Se trata también de la prioridad de los intereses colectivos sobre los individuales en cuanto al trabajo que se realiza para la comunidad" (p.96).

Para otras líderes, el tema de liderazgo y su significado está relacionado también con el tema del poder, para lo cual Fisher (1992), citado por Montero (2006), afirma que "el poder consiste en la capacidad de un individuo de orientar la acción de otro" (p.39), lo que de entrada identifica una posición en la cual las personas pueden elegir y dirigir sus actuaciones libremente. Con ello y en el contexto del presente trabajo, es importante resaltar que en las líderes se identificaron diversos tipos de poder, uno de ellos de tipo informativo, categoría creada por French y Raven (1959), citados por Montero (2006), el cual se deriva de la capacidad de transmitir información a los otros y de orientarlos en relación a la misma, lo cual es importante para las líderes porque a partir de él pueden orientar a otros desde sus propias necesidades e intereses, identificándola como alguien que sabe lo que a ellos les interesa; ese saber algo, por sencillo que sea, es un recurso que genera poder.

De Crespigny, citado en Ibáñez (1980) y retomado por Montero (2006) en consonancia con este tema, añade otra categoría y es el "poder reactivo", centrado en generar reacciones de manera planificada e intencional, en orientar la conducta de los demás y aportar al logro de objetivos específicos. Al considerar esto, las líderes también ejercen dicho poder en tanto sus actuaciones no son al azar, sino que son pensadas teniendo en cuenta los intereses de las personas y sus necesidades, propiciando su autonomía para hacer elecciones y tomar decisiones que les deje capacidad de agencia, logrando convertir un recurso en acciones importantes para su vida, desarrollar capacidades y que éstas puedan ser expresadas en funcionamientos.

Interpretar los significados construidos por las líderes en relación a su rol, brinda entonces una pauta para identificar sus aportes o contribuciones a la superación de la pobreza extrema y generar reflexiones al respecto; bajo este panorama, es importante mencionar como en el marco de la presente investigación, lejos de concebirse la pobreza sólo como la falta de ingresos, se concibe es como la privación de las capacidades. Para Amartya Sen (2000), "Lo que hace la perspectiva de las capacidades en el análisis de la pobreza es contribuir a comprender mejor la naturaleza y las causas de la pobreza y la privación, trasladando la atención principal de los medios (y de un determinada medio que suele ser objeto de una atención exclusiva, a saber, la renta) a los fines que los individuos tienen razones para perseguir y, por lo tanto, a las libertades necesarias para poder satisfacer estos fines". (p.7)

Al considerar lo anterior, si bien para las líderes el tema de las rentas/ingresos son importantes porque dan respuesta a algunas de sus necesidades inmediatas como alimentación y estudio que son básicamente las razones por las que reciben un incentivo económico, para éstas, esto realmente no es una prioridad o exclusividad para alcanzar el bienestar a través del grado de libertad que los seres humanos tienen para elegir. De éste modo, éstas hicieron hincapié fue en el hecho de que si bien las personas tienen la libertad de elegir entre distintas formas de vida, un aspecto al que le deben apuntar desde su perspectiva es indiscutiblemente al tema formativo, a través del cual se pueden desarrollar las capacidades y alcanzar el bien-estar a nivel individual y colectivo, no en vano que para ellas una de las mayores contribuciones que realizan al programa sobre el tema de la pobreza sea capacitarse, cualificarse y mantener informada a la población.

Es así, como el papel multiplicador de las líderes es fundamental ya que no sólo potencia habilidades propias, sino que informa a los titulares y comunidad en general, permitiéndoles adquirir nuevos conocimientos relacionados con distintos temas de bienestar comunitario, de allí que sea tan importante participar en la vida de la comunidad, no sólo porque 
reduce la exclusión, sino porque permite fomentar la democracia, potenciar la toma de decisiones y disminuir las conductas reproductivas de la pobreza, aumentando su calidad de vida y libertad.

En relación al tema, se visualiza la importancia de una intervención del Estado, especialmente en el desarrollo de Políticas Públicas mediante las cuales se garanticen beneficios a la población. En ésta materia entra a jugar el Programa Más Familias en Acción, que más allá de otorgar un incentivo económico, también exige una corresponsabilidad por parte de las personas, en las que éstas se movilicen a cumplir unos compromisos en materia de salud y educación, evidenciando que es necesario que cada individuo emprenda acciones que favorezcan su desarrollo y que por ende les ayude a avanzar en su situación actual. Es así, como a través de la figura de liderazgo se fomenta que la familia como núcleo central de la sociedad se empodere de su propia vida, sea autogestora de su proceso, agente de cambio de la sociedad y de su propia vida, en otras palabras, que expanda su capacidad de agencia, dejando de un lado la visión del individuo como receptáculo o agente pasivo, sino como sujeto activo, libre para hacer aquello que tiene razones para valorar y convertir los recursos en pro de su bienestar.

Con ello, se busca que líderes y titulares sean autónomos y cuenten con la habilidad suficiente de elegir y tomar decisiones sobre sus propias vidas; es allí, donde aparece justamente el tema de las capacidades, de tal forma que éstas posibiliten alcanzar aquello que es valioso para cada quien, logrando convertir los medios en bien-estar y expandir su libertad real, siendo ésta última un eficiente medio para alcanzar el desarrollo.

\section{CONCLUSIONES}

Adentrarse en procesos de liderazgo asumidos desde la figura femenina, posibilita identificar cómo las mujeres han ido ganando cada vez más un espacio y reconocimiento en el ámbito público y reivindicar los procesos de liderazgo como asuntos necesarios en toda sociedad, en donde se hace vital contar con una figura que oriente acciones de la vida comunitaria, impulsada por el convencimiento de que es posible movilizar a otros en la consecución de metas comunes y de ver en la participación un mecanismo para alcanzar el desarrollo.

La puesta en escena y asunción de éste rol genera grandes contribuciones en temas como la pobreza extrema, en el que se evidenció en las líderes una apuesta en términos del desarrollo de capacidades a través de procesos formativos y educativos que aporten a la libertad e incidan en el logro del bien-estar. En este sentido, se actúa bajo la premisa de que la expansión de la libertad propicia el desarrollo de capacidades, que la existencia de programas sociales conlleva a la inclusión y que la autonomía es un elemento esencial en tanto posibilita elegir y tomar decisiones sobre la vida.

Estas premisas, además de aportar al cambio de paradigmas en relación con la pobreza, hacen hincapié en la necesidad de que la figura de liderazgo continúe fortaleciéndose en compañía de programas sociales como Medellín Solidaria - Más Familias en Acción, apostándole a uno de los Objetivos del Milenio y dejando capacidad instalada en las personas, en donde ellas puedan despleglar todas sus potencialidades y capacidad de agencia, desarrollando la conciencia de que es posible ser y actuar en pro del mejoramiento de la libertad, del bienestar y por ende del Desarrollo Humano.

\section{RECOMENDACIONES}

Abordar temas como el que es objeto de la presente investigación, hace surgir dos recomendaciones importantes: la primera, centrada en que la institucionalidad genere más acciones a partir de las cuales se fortalezca el tema de liderazgo en la ciudad de Medellín, a través del cual sea posible movilizar a 
otros y adquirir conocimientos que puedan extrapolarse al entorno social, aportando a la transformación de la ciudad; la segunda, enfocada en continuar realizando investigaciones de este tipo, en donde se aborde el liderazgo desde las personas que participan en los procesos, esto implica protagonistas, técnicosprofesionales, coordinadores de programas, quienes desde su lugar pueden brindar nuevas visiones y aportar nuevos elementos que lleven a su consolidación en el territorio.

El estudio hace emerger entonces nuevas preguntas de investigación, que deben ser respondidas mediante otros procesos, por ejemplo: ¿Desde el enfoque de la libertad será necesario construir nuevos indicadores cualitativos de desarrollo? ¿Los modelos de liderazgo femenino son válidos solamente en contextos de programas de desarrollo? ¿El liderazgo femenino en programas de desarrollo ha contribuido a disminuir la violencia en el contexto actual? ¿Cuál es la importancia del liderazgo femenino desde el enfoque de las capacidades para las futuras generaciones?

\section{BIBLIOGRAFÍA}

DE CRESPIGNY, citado en Ibáñez (1980) y retomado por Montero, M. (2006). En: Teoría y práctica de la psicología comunitaria: La tensión entre comunidad y sociedad [en línea] Buenos Aires, ed. Paidós.

FISHER (1992), citado por Montero, M. (2006). En: Teoría y práctica de la psicología comunitaria: La tensión entre comunidad y sociedad, [en línea], Buenos Aires, ed. Paidós.

FRENCH Y RAVEN (1959), citados por Montero, M. (2006). En: Teoría y práctica de la psicología comunitaria: La tensión entre comunidad y sociedad [en línea], Buenos Aires, ed. Paidós.

GALEANO, E. (2004). Estrategias de investigación social cualitativa: el giro de la mirada, Colombia, ed. La carreta editores.

GOUGH, I. (2007/08) “El enfoque de las capacidades de
M. Nussbaum: Un análisis comparado con nuestra teoría de las necesidades humanas", Papeles de Relaciones Ecosociales y Cambio Global, no 100 , CIP-Ecosocial/Icaria, Madrid.

KAWULICH, B. (2005). La observación participante como método de recolección de datos. Forum: Qualitative Social Research. Mayo. Volumen 6, N ${ }^{\circ}$ 2, Artículo 43.

MANNEN, V (1990) citado por Sandoval, C. (2002). En: Investigación social cualitativa, BogotáColombia, ARFO Editores e Impresores Ltda.

Manual Operativo Versión 1 Más Familias en Acción. Dirección de Ingreso Social, 2013 [consultado el 01 de Julio del 2013]. Disponible en internet: http:// www.dps.gov.co/documentos/8122 Manual operativo programa_M\% $3 \% \mathrm{~A} 1 \mathrm{~s}$ _Familias en Acci $\% \mathrm{C} 3 \% \mathrm{~B} 3 \mathrm{n} . \mathrm{pdf}$

MARSHALL y ROSSMAN (1989), citados por Kawulich, B. (2005). En: La observación participante como método de recolección de datos. Forum: Qualitative Social Research. Mayo. Volumen 6, $\mathrm{N}^{\mathrm{o}}$ 2, Artículo 43.

MEJÍA, R y SANDOVAL, S. (1998). Tras las vetas de la investigación cualitativa. Perspectivas y acercamientos desde la práctica, México, ed. TESO.

MONTERO, M. (2006). Teoría y práctica de la psicología comunitaria: La tensión entre comunidad y sociedad, Buenos Aires, ed. Paidós.

MOSCOVICI, S citado por Materán, A. (2008). En: "Las representaciones sociales: Un referente teórico para la investigación educativa", Geoenseñanza, Venezuela, vol. 13, núm. 2, julio-diciembre, pp. 243-248, Universidad de los Andes.

NUSSBAUM, M citada por Gough, I. (2007/08) En: "El enfoque de las capacidades de M. Nussbaum: Un análisis comparado con nuestra teoría de las necesidades humanas", Papeles de Relaciones Ecosociales y Cambio Global, $\mathrm{n}^{\mathrm{o}}$ 100, CIPEcosocial/Icaria, Madrid.

PÉREZ, E y SÁNCHEZ, J. (2005) “La educación comunitaria: Una concepción desde la Pedagogía de 
la Esperanza de Paulo Freire", Revista Venezolana de Ciencias Sociales, UNERMB, Vol. 9 No. 2, Venezuela.

RAY, M. (2003). La riqueza de la fenomenología: preocupaciones filosóficas, teóricas $\mathrm{y}$ metodológicas. En: Morse J. Asuntos Críticos en los métodos de investigación cualitativa, MedellínColombia, Universidad de Antioquia, p 139-157.

RISO, W. (2006). Terapia Cognitiva: Fundamentos teóricos y conceptualización del caso clínico, Bogotá - Colombia, Grupo Editorial Norma.

SANDOVAL, C. (2002). Investigación Social Cualitativa, Bogotá- Colombia, ARFO Editores e Impresores Ltda.

SEN, A. (2000). Desarrollo y Libertad. Capítulo 4: La Pobreza como privación de capacidades, Buenos Aires, ed. Planeta S.A, p. 114-141.

Sistematización del Componente Grupal del Programa
Medellín Solidaria- Más Familias en Acción, Medellín - Colombia, 2011. [Documento digital].

TORRES, A. (2011). Educación Popular: Trayectoria y Actualidad, Dirección General de Producción $y$ Recreación de Saberes, Venezuela, imprenta Universitaria UVB.

VALLES, M. (2006). Técnicas investigativas de investigación social: Reflexión metodológica y práctica profesional, Perú, ed. LIBERATI.

VASCO, C. (1990). Tres estilos de trabajo en las ciencias sociales, Bogotá, CINEP.

WOODS (1987), referenciado en: La investigación etnográfica [en línea]. [Consultado el 22 de Julio de 2014]. Disponible en internet: http://html. rincondelvago.com/investigacion-etnografica.html YIN, R. (1994). Investigación sobre estudio de casos: Diseño y método, ed SAGE, London. 